Reasons for AZA/MP discontinuation were intolerance to therapy (38\%), and failure of therapy (62\%) despite dose optimisation.

Steroid-free remission was achieved in 11 patients (52\%), and this appears sustained at 12 months follow-up. A further 4 patients (19\%) report improved symptoms with MTX, but remain dependent on low-dose steroids (although it is noted that 1 of these patients has co-existent rheumatoid arthritis which may explain this). MTX was discontinued in 4 patients (19\%) because of a lack of clinical response ( $\mathrm{n}=$ $1)$, side-effects $(n=2)$ or planned pregnancy $(n=1)$. Sideeffects reported with MTX were liver toxicity and skin rashes. A final 2 patients (10\%) have shown promising results with MTX but are not yet eligible for 12-month follow-up. Of the 21 patients included, 20 remain on oral therapy, and one has switched to parenteral MTX. Of note, none of the MTX patients have progressed to colectomy, in contrast to previous studies.

Conclusion Our study has shown good efficacy with MTX, with approximately half of UC patients achieving steroid-free clinical remission at 12 months. In contrast to previous studies, our experience suggests it is a useful treatment option in patients previously failing or intolerant of optimised thiopurine therapy. Disclosure of Interest None Declared.

\section{PTU-072 OUTCOMES OF THE USE OF INFLIXIMAB AND ADALIMUMAB IN PATIENTS WITH CROHN'S DISEASE AT A DISTRICT GENERAL HOSPITAL}

C Lane*, S Ramakrishnan, J Dougherty. Gastroenterology, Warrington Hospital, Warrington, UK

\subsection{6/gutjnl-2014-307263.146}

Introduction Infliximab (IFX) and adalimumab (ADA) are licensed for the treatment of severe Crohn's disease (CD). NICE recommends patients should receive these agents as a planned course of treatment for 12 months or until treatment failure. Patients should have their disease reassessed to determine if they have active disease and whether ongoing therapy is appropriate. We assessed our adherence to the NICE recommendations and present our outcomes for patients who had treatment discontinued following remission.

Methods Patients who had received treatment with IFX and/or ADA from Jan 2011 to Sep 2013 were identified from a database held by our pharmacy. Data was collected from patient case notes and a database of clinic letters. A total of 49 patients were identified. Cases were assessed for adherence to NICE recommendations.

Results 24 patients had been on IFX only (49\%), 14 patients on ADA only (28.6\%) and 11 started on IFX then switched to ADA (22.4\%). 8 patients had evidence of fistulating disease. Mean age was 39 years (range 17-60). All patients (100\%) had severe active CD that did not respond to conventional therapy (79.6\%) or were intolerant or had contraindications $(20.4 \%)$ to therapy. All patients (100\%) were reassessed to determine whether ongoing treatment was still clinically appropriate. All patients $(100 \%)$ were treated and reviewed by clinicians with experience in their use. Discussion regarding risks and benefits of continued treatment occurred in $77.8 \%$ of cases. 39 patients $(79.6 \%)$ had been on treatment for greater than 1 year. Of these, 20 patients had treatment discontinued (51.3\%); 3 were due to a reaction/ intolerance, 10 were due to treatment failure, and 7 due to deep clinical remission (17.9\%). 2 patients (28.6\%) had disease recurrence following treatment withdrawal due to remission. Mean time to relapse following withdrawal was 9 months (range 3-15). Of all patients found to be in deep clinical remission (n $=12,30.8 \%)$ after 12 months of treatment, 6 patients had treatment continued partly due to patient choice (66.7\%). Patients who continue treatment all had their disease reassessed at least every 12 months.

Conclusion There is good adherence to NICE guidelines in our cohort, however despite evidence of deep clinical remission some patients declined to have treatment discontinued partly due to anxiety of relapse. Our relapse rate following withdrawal of biological treatment appears lower than that found in the literature.

\section{REFERENCES}

NICE Technology Appraisal Guidance 187: infliximab (review) and adalimumab for the treatment of Crohn's disease

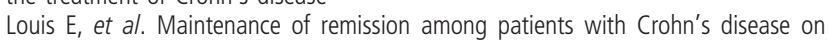
antimetabolite therapy after infliximab therapy is stopped. Gastroenterology 2012;142:63-70

Disclosure of Interest None Declared.

\section{PTU-073 COMPARISON OF SICUS VERSUS MR-ENTEROGRAPHY IN PATIENTS WITH CROHN'S DISEASE}

${ }^{1} \mathrm{C}$ Alexakis*, ${ }^{1} \mathrm{~V}$ Chhaya, ${ }^{2} \mathrm{D}$ Tzias, ${ }^{2} \mathrm{~J}$ Pilcher, ${ }^{1} \mathrm{R}$ Pollok. ${ }^{1}$ Department of Gastroenterology, St George's Hospital, London, UK; ${ }^{2}$ Department of Radiology, St George's Hospital, London, UK

\subsection{6/gutjnl-2014-307263.147}

Introduction Small intestinal contrast-enhanced ultrasonography (SICUS) is an emerging radiological technique for the imaging of patients with Crohn's disease that avoids exposure to diagnostic medical radiation. We have previously demonstrated that SICUS is diagnostically comparable to small bowel barium follow through and computerised tomography in the diagnosis of Crohn's ${ }^{1}$ MR enterography (MRE) is becoming the gold standard radiological technique for the diagnosis of complications in patients with Crohn's, but is expensive and access is limited. We aimed to compare the diagnostic sensitivity of SICUS with MRE in routine clinical practice.

Methods Patients with established Crohn's disease, who had undergone both SICUS and MRE within 6 months of each other were identified retrospectively from the radiology database at a UK tertiary centre. Imaging and reports were reviewed for both modalities. Kappa coefficient data was calculated for luminal parameters including the presence of strictures, stricture number and location, the presence of abscess/fistulae, mucosal thickening, active mucosal inflammation and fibrotic changes. Reported stricture lengths were compared using paired student's $t$-test. Inflammatory markers including platelet levels, where available, were recorded within 2 weeks of each of the imaging modalities as a surrogate marker for active inflammation.

Results 20 Crohn's patients were identified (10 male), with a mean age of 30.1 years at time of first investigation. Mean time between modalities was 72.3 days (range 2-147). There was no significant difference between mean platelet counts between the 2 radiological tests. Agreement between the 2 modalities was excellent for the presence of stricturing disease $(k=0.92,95 \%$ CI 0.71-1.00), stricture number $(\mathrm{k}=0.91,95 \%$ CI $0.73-1.00)$ and stricture location $(\mathrm{k}=0.91,95 \%$ CI $0.71-1.00)$. Agreement was good for the presence of fistulae $(\mathrm{k}=0.74,95 \%$ CI 0.40 1.00) and mucosal thickening ( $\mathrm{k}=0.74,95 \%$ CI $0.40-1.00)$. 